\title{
Identification of Individuals with MCI via Multimodality Connectivity Networks
}

\author{
Chong-Yaw Wee ${ }^{1}$, Pew-Thian Yap ${ }^{1}$, Daoqiang Zhang ${ }^{1}$, \\ Kevin Denny ${ }^{2}$, Lihong Wang ${ }^{2}$, and Dinggang Shen ${ }^{1}$ \\ 1 Department of Radiology and Biomedical Research Imaging Center (BRIC), \\ University of North Carolina at Chapel Hill, NC, USA \\ 2 Brain Imaging and Analysis Center (BIAC), \\ Duke University Medical Center, Durham, NC, USA
}

\begin{abstract}
Mild cognitive impairment (MCI), often an early stage of Alzheimer's disease (AD), is difficult to diagnose due to the subtlety of cognitive impairment. Recent emergence of reliable network characterization techniques based on diffusion tensor imaging (DTI) and restingstate functional magnetic resonance imaging (rs-fMRI) has made the understanding of neurological disorders at a whole-brain connectivity level possible, providing new avenues for brain classification. Taking a multi-kernel SVM, we attempt to integrate these two imaging modalities for improving classification performance. Our results indicate that the multimodality classification approach performs better than the single modality approach, with statistically significant improvement in accuracy. It was also found that the prefrontal cortex, orbitofrontal cortex, temporal pole, anterior and posterior cingulate gyrus, precuneus, amygdala, thalamus, parahippocampal gyrus and insula regions provided the most discriminant features for classification, in line with the results reported in previous studies. The multimodality classification approach allows more accurate early detection of brain abnormalities with larger sensitivity, and is important for treatment management of potential AD patients.
\end{abstract}

\section{Introduction}

Alzheimer's disease (AD) is one of the most prevalent dementia in older adults worldwide, characterized by cognitive and intellectual deficits that is serious enough to interfere daily life. Accurate diagnosis of AD is crucial for early treatment. Mild cognitive impairment (MCI), often an early stage of AD, is a good target for early diagnosis and therapeutic interventions of AD. Nevertheless, MCI is difficult to diagnose due to the subtlety of cognitive impairment.

Recently, several imaging modalities have been proven to be effective in AD and MCI diagnosis, including diffusion tensor imaging (DTI) 6], magnetic resonance imaging (MRI) 9], resting-state functional MRI (rs-fMRI) 8] and positron emission tomography (PET) [5]. Nevertheless, most existing pattern classification methods use only each of these modalities independently for AD and MCI

G. Fichtinger, A. Martel, and T. Peters (Eds.): MICCAI 2011, Part II, LNCS 6892, pp. 277-284, 2011.

(C) Springer-Verlag Berlin Heidelberg 2011 
diagnosis. More effort should be made in integrating of two or more modalities since combining complementary information of different biomarkers can be useful for improving diagnosis.

To the best of our knowledge, DTI and rs-fMRI have not been combined at a network level for identifying individuals with MCI, although they have been employed separately with reasonably good classification performance. The current study is the first attempt to integrate these two modalities to identify individuals with MCI from normal controls. We seek to validate whether complementary structural and functional information can be combined to improve classification performance. We will also report brain regions that contribute most to the classification performance. While confirming findings of previous studies, this paper sheds new light on the effectiveness of applying multimodality information for diagnosis of progressive neurodegenerative disorders.

\section{Materials and Methods}

The current study involved 27 participants, 10 individuals with MCI and 17 socio-demographically matched healthy controls. Informed consent was obtained from all participants, and the experimental protocols were approved by the institutional ethics board. Confirmation of diagnosis for all subjects was made via expert consensus panels. Demographic information of the participants is shown in Table 1.

Table 1. Demographic information of the participants involved in this study. Group difference was assessed using two-sample $t$-tests.

\begin{tabular}{|l|c|c|c|}
\hline Group & MCI & Normal & $p$-value \\
\hline \hline No. of subjects & 10 & 17 & - \\
Gender & $5 \mathrm{M} / 5 \mathrm{~F}$ & $8 \mathrm{M} / 9 \mathrm{~F}$ & - \\
Age (mean $\pm \mathrm{SD})$ & $74.2 \pm 8.6$ & $72.1 \pm 8.2$ & 0.5372 \\
Years of education $($ mean $\pm \mathrm{SD})$ & $17.7 \pm 4.2$ & $16.3 \pm 2.4$ & 0.2804 \\
MMSE (mean $\pm \mathrm{SD})$ & $28.4 \pm 1.5$ & $29.4 \pm 0.9$ & 0.0405 \\
\hline
\end{tabular}

\subsection{Data Acquisition}

Data acquisition was performed using a 3.0-Tesla GE Signa EXCITE scanner. Diffusion-weighted images of each participant were acquired with 25-direction diffusion-weighted whole-brain volumes with the following parameters: $b=0$, $1000 \mathrm{~s} / \mathrm{mm}^{2}$, flip angle $=90^{\circ}, \mathrm{TR} / \mathrm{TE}=17000 / 78 \mathrm{~ms}, 72$ slices, imaging matrix $=128 \times 128, \mathrm{FOV}=256 \times 256 \mathrm{~mm}^{2}$, resulting in a voxel dimension of $2 \times 2 \times 2 \mathrm{~mm}^{3}$ reconstructed resolution. Resting-state functional images were acquired using a SENSE inverse-spiral pulse sequence with the following parameters: flip angle $=77^{\circ}, \mathrm{TR} / \mathrm{TE}=2000 / 32 \mathrm{~ms}, 34$ slices, imaging matrix $=64 \times 64, \mathrm{FOV}=$ $256 \times 256 \mathrm{~mm}^{2}$, resulting in a voxel resolution of $4 \times 4 \times 4 \mathrm{~mm}^{3}$. All the subjects were told to keep their eyes open and stare at a fixation cross in the middle of the screen during scanning, which lasted for 5 minutes. 


\subsection{Data Post-processing and Network Construction}

The DTI images were first parcellated into 90 regions by propagating the automated anatomical labeling (AAL) ROIs 11] to each image using a deformable DTI registration algorithm. Whole-brain streamline fiber tractography was then performed on each image using ExploreDTI 7] with the following parameters: starting/stopping $\mathrm{FA}=0.45 / 0.25$, minimum/maximum fiber length $=$ $20 / 400 \mathrm{~mm}$. The number of fibers passing through each pair of regions was counted. Two regions were considered as anatomically connected if fibers passing through their respective masks were present, giving us connection topology of the network. On top of the fiber count based connectivity network, averages of on-fiber fractional anisotropy (FA), mean diffusivity (MD) and principal diffusivity values were computed to form another 5 connectivity networks with the same topology but conveying different biophysical properties. Examples of the constructed connectivity maps are shown in Figure 1.
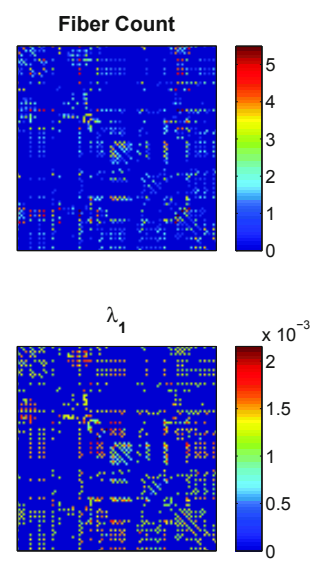
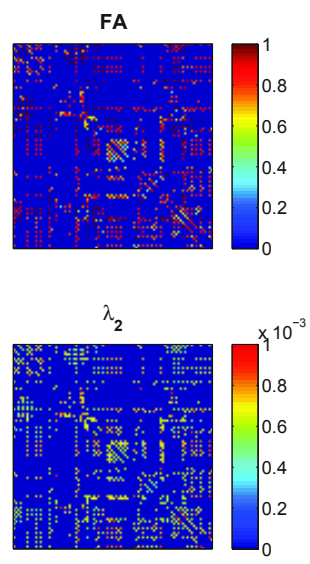
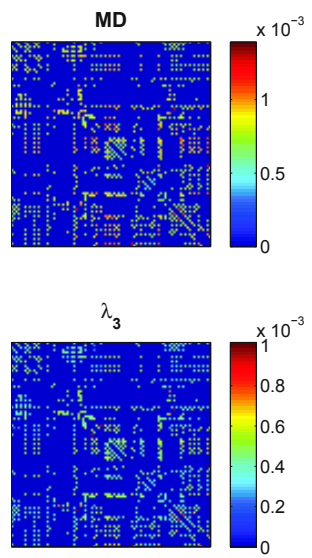

Fig. 1. Connectivity maps constructed from DTI different physiological measures

Post-processing of the fMRI images, such as slice timing correction and headmotion correction were performed using the Statistical Parametric Mapping (SPM8, http://www.fil.ion.ucl.ac.uk.spm) software package. The images were then masked with their respective gray matter (GM) masks, which were created by segmenting the GM regions from their T1-weighted images. This eliminated the white matter and cerebrospinal fluid from contributing to the fMRI time series, which contain a relatively high proportion of noise caused by cardiac and respiratory cycles 12 .

Then, we parcellated the brain space into 90 ROIs by warping the fMRI images to the AAL template. For each subject, the mean time series was computed for each GM-masked region. Temporal band-pass filtering of frequency interval $(0.025 \leq f \leq 0.100 \mathrm{~Hz})$ was then performed to minimize the effects of lowfrequency drift and high-frequency noise since the fMRI dynamics of neuronal 
activities are most salient within this frequency interval. This frequency interval was further decomposed into 5 equally divided, non-overlapping frequency subbands, enabling a relatively frequency specific analysis of the regional mean time series via a multi-spectral characterization.

Functional connectivity, which examines interregional correlations in neuronal variability, was measured using pairwise Pearson correlation coefficients between the ROI pairs. Given a set of $N$ random variables, the Pearson correlation matrix is a symmetric matrix in which each off-diagonal element is the correlation coefficient between a pair of variables. We considered the brain regions as a set of nodes and the correlation coefficients as signed weights on the set of edges. Fisher's $r$-to- $z$ transformation was applied on the elements of the Pearson correlation matrix to improve the normality of Pearson correlation coefficients. Examples of the constructed functional connectivity maps for a normal control (NC) and an MCI patient are shown in the top and bottom rows of Figure 2, respectively.
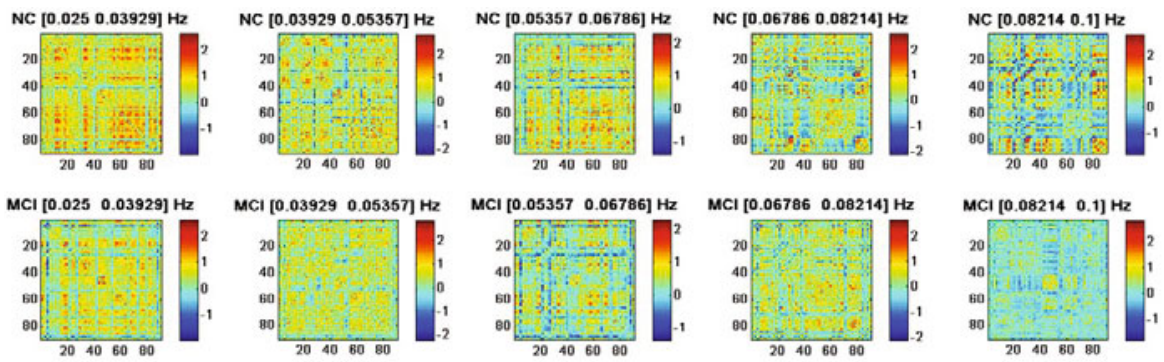

Fig. 2. Multi-spectral functional connectivity maps for NC (top) and MCI (bottom), respectively

\subsection{Multi-Kernel SVM Multimodality Classification}

The proposed framework for integrating DTI and rs-fMRI is divided into 3 stages: feature extraction, feature selection and multimodality data fusion. In the first stage, the weighted local clustering coefficient, a measure that quantifies the cliquishness of the nodes, is extracted from all connectivity maps as

$$
f(p)=\frac{\sum_{q: q \neq p \in \zeta} 2 t(p, q)}{k_{p}\left(k_{p}-1\right)},
$$

where $\zeta$ is the subnetwork comprising of $k_{p}$ nodes directly connected to the $p$-th node, and $t(p, q)$ is the edge weight between the $p$-th node and $q$-th node. Hence, a total of 90 features can be obtained from each connectivity map, producing for each subject a pool of 540 and 450 features for DTI and rs-fMRI, respectively.

In the second stage, statistical $t$-test was performed to select features for classification. Features with $p$-values smaller than a predefined threshold will be selected from each individual modality before they were integrated using a 
multi-kernel SVM algorithm. In the third stage, for $n$ training samples with each of them is of $M$ modalities, the multi-kernel SVM solves the following primal problem

$$
\begin{aligned}
& \min _{\mathbf{w}^{(m)}, b, \xi_{i}} \frac{1}{2} \sum_{m=1}^{M} \beta_{m}\left\|\mathbf{w}^{(m)}\right\|^{2}+C \sum_{i=1}^{n} \xi_{i}, \\
& \text { s.t. } y_{i}\left[\sum_{m=1}^{M} \beta_{m}\left(\left(\mathbf{w}^{(m)}\right)^{T} \phi^{(m)}\left(\mathbf{X}_{i}^{(m)}\right)+b\right)\right] \geq 1-\xi_{i} ; \text { with } \xi_{i} \geq 0, i=1, \ldots, n
\end{aligned}
$$

with $\mathbf{X}_{i}^{(m)}=\left\{\mathbf{x}_{i, 1}^{(m)}, \ldots, \mathbf{x}_{i, D}^{(m)}\right\}$ denotes a feature vector of the $m$-th modality of the $i$-th sample $\left(D=\right.$ number of maps in the $m$-th modality, $\mathbf{x}_{i, d}^{(m)}=$ $\left\{f_{i, d}^{(m)}(1), \ldots, f_{i, d}^{(m)}(90)\right\}$ and $\left.d=1, \ldots, D\right)$ and $y_{i} \in\{-1,1\}$ as its corresponding class label. Parameters $\xi_{i}, C, b, \mathbf{w}^{(m)}, \phi^{(m)}$ and $\beta_{m} \geq 0$ denote the distance of the $i$-th misclassified observation from its correct side of the margin, the model parameter that controls for the amount of constraint violations introduced by $\xi_{i}$, the bias term, the normal vector of hyperplane, the kernel-induced mapping function and the weighting factor of the $m$-th modality, respectively.

The dual form of multi-kernel SVM is solved as

$$
\begin{array}{ll}
\max _{\alpha} & \sum_{i=1}^{n} \alpha_{i}-\frac{1}{2} \sum_{i, j} \alpha_{i} \alpha_{j} y_{i} y_{j} \sum_{m=1}^{M} \beta_{m} k^{(m)}\left(\mathbf{X}_{i}^{(m)}, \mathbf{X}_{j}^{(m)}\right), \\
\text { s.t. } & \sum_{i=1}^{n} \alpha_{i} y_{i}=0 ; \text { with } 0 \leq \alpha_{i} \leq C, i=1, \ldots, n
\end{array}
$$

where $\alpha_{i}$ is the Lagrange multiplier and $k^{(m)}\left(\mathbf{X}_{i}^{(m)}, \mathbf{X}_{j}^{(m)}\right)=\phi^{(m)}\left(\mathbf{X}_{i}^{(m)}\right)$ $\phi^{(m)}\left(\mathbf{X}_{j}^{(m)}\right)$ is the kernel function for a pair of training samples of the $m$-th modality.

Given a new test sample $\mathbf{X}=\left\{\mathbf{X}^{(1)}, \ldots, \mathbf{X}^{(M)}\right\}$ and let the kernel between the new test sample and each training sample of the $m$-th modality be $k^{(m)}\left(\mathbf{X}_{i}^{(m)}\right.$, $\left.\mathbf{X}^{(m)}\right)=\phi^{(m)}\left(\mathbf{X}_{i}^{(m)}\right) \phi^{(m)}\left(\mathbf{X}^{(m)}\right)$, the decision function for the predicted label can be determined as

$$
F(\mathbf{X})=\operatorname{sign}\left(\sum_{i=1}^{n} y_{i} \alpha_{i} \sum_{m=1}^{M} \beta_{m} k^{(m)}\left(\mathbf{X}_{i}^{(m)}, \mathbf{X}^{(m)}\right)+b\right)
$$

The multi-kernel SVM can be naturally embedded into the conventional single kernel SVM by interpreting $k\left(\mathbf{X}_{i}, \mathbf{X}\right)=\sum_{m} \beta_{m} k^{(m)}\left(\mathbf{X}_{i}^{(m)}, \mathbf{X}^{(m)}\right)$ as a mixed kernel between the multimodality training sample $\mathbf{X}_{i}$ and the test sample $\mathbf{X}$. A linear kernel SVM classifier based on the LIBSVM library [1] was employed to demonstrate that the improvement obtained is due mainly to the complementary information of different modalities. 


\section{Experimental Results}

The proposed multi-kernel SVM based multimodality classification approach was compared with the single modality approach and the direct data fusion method. In the single modality approach, only features selected from a single imaging modality (DTI or rs-fMRI) were applied for SVM classifier training. While in the direct data fusion method, all 990 features, which including the DTI and rsfMRI features, were first concatenated into a long vector before feature selection using the $t$-test. In the multi-kernel approach, the optimal weighting factor, $\beta_{m}$, and SVM parameter, $C$, were determined via grid search over a fixed range. In all compared approaches, the $p$-value of 0.01 was used for each training set during cross-validation. Classification was performed based on the $z$-scores of the features.

We employed various measures to evaluate the diagnostic power of the compared methods. The Youden's index, Balanced ACcuracy (BAC) and F-score are defined respectively as 10 .

$$
\begin{aligned}
\mathbf{Y} & =\text { Sensitivity }+ \text { Specificity }-1=\frac{\mathrm{TP}}{\mathrm{FP}}+\frac{\mathrm{TN}}{\mathrm{FN}}-1, \\
\mathbf{B A C} & =\frac{1}{2} \times\left[\frac{\mathrm{TP}}{\mathrm{TP}+\mathrm{FN}}+\frac{\mathrm{TN}}{\mathrm{TN}+\mathrm{FP}}\right], \\
\mathbf{F} & =2 \times\left[\frac{\text { precision } \times \text { recall }}{\text { precision }+ \text { recall }}\right],
\end{aligned}
$$

where

$$
\text { precision }=\frac{\mathrm{TP}}{\mathrm{TP}+\mathrm{FP}} ; \text { recall }=\frac{\mathrm{TP}}{\mathrm{TP}+\mathrm{FN}}
$$

with TP, TN, FP and FN denoting the true positive, true negative, false positive and false negative, respectively.

Comparison was performed via leave-one-out cross-validation due to limited number of available samples. Classification performance for individuals with MCI using single and multimodality connectivity networks are summarized in Table 2 The proposed method yields a classification accuracy of $96.59 \%$, which is an increment of at least $7.41 \%$ from that of the single modality approach and the direct data fusion method.

Table 2. Classification performance of single and multimodality connectivity networks. $(\mathrm{ACC}=$ ACCuracy; SEN = SENsitivity; SPE $=$ SPEcificity; Youden $=$ Youden's index $)$

\begin{tabular}{|l|c|c|c|c|c|c|c|}
\hline Method & ACC (\%) & SEN(\%) & SPE(\%) & AUC & Youden & BAC & F-score \\
\hline \hline fMRI & 55.56 & 60.00 & 52.94 & 0.6059 & 0.1294 & 0.5647 & 0.5000 \\
DTI & 88.89 & 80.00 & 94.12 & 0.9353 & 0.7412 & 0.8706 & 0.8421 \\
Direct & 88.89 & 90.00 & 88.24 & 0.9118 & 0.7824 & 0.8912 & 0.8571 \\
kernel & 96.30 & 100.00 & 94.12 & 0.9529 & 0.9412 & 0.9524 & 0.9706 \\
\hline
\end{tabular}




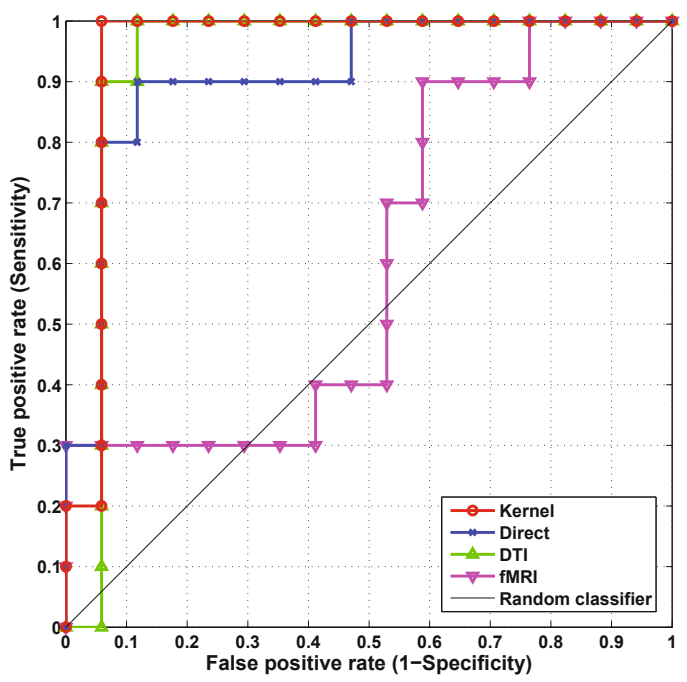

Fig. 3. ROC curves.

A cross-validation estimation of the generalization performance shows an area of 0.9529 under the receiver operating characteristic (ROC) curve (AUC), indicating excellent diagnostic power. ROC curves for all compared methods are shown in Figure 3 .

The most discriminant regions that were selected in the course of the classification include the prefrontal cortex and orbitofrontal cortex [5], temporal pole 4], anterior and posterior cingulate gyrus, precuneus and insula [3], amygdala [2], thalamus [13], parahippocampal gyrus [8], in line with results reported in previous studies. The discriminative regions were selected based on whether their clustering coefficients were selected to be employed for classification. The order of the regions is determined by their $p$-values; a smaller $p$-value indicates higher discriminative power.

\section{Discussions and Conclusion}

We investigated the diagnostic power of multimodality classification of the DTI and rs-fMRI connectivity maps in identifying individuals with MCI. In this framework, linear SVM classifiers were trained using a mixed kernel that was constructed from the individual kernels of multiple modalities. This framework shows better classification performance, and justifies the hypothesis that the DTI and rs-fMRI contain complementary information, each of them indispensable particularly for achieving better diagnostic power. Furthermore, the higher sensitivity rate of the proposed approach is an important improvement since the cost of misclassifying individuals with MCI is significantly larger than that of misclassifying normal controls. Correct diagnosis of individuals with MCI enables early treatment management of potential AD patients possible and thus reduces the MCI to $\mathrm{AD}$ conversion rate. The promising results indicate that the 
proposed framework can provide an alternative and complementary approach for clinical diagnosis of brain degeneration.

\section{References}

1. Chang, C.C., Lin, C.J.: LIBSVM: a library for support vector machines (2001), software, available at http://www.csie.ntu.edu.tw/ cjlin/libsvm

2. Dai, W., Lopez, O.L., Carmichael, O.T., Becker, J.T., Kuller, L.H., Gach, H.M.: Mild cognitive impairment and Alzheimer disease: Patterns of altered cerebral blood flow at MR imaging. Radiology 250, 856-866 (2009)

3. Davatzikos, C., Bhatt, P., Shaw, L.M., Batmanghelich, K.N., Trojanowski, J.Q.: Prediction of MCI to AD conversion, via MRI, CSF biomarkers and pattern classification. Neurobiol Aging (2010)

4. Flavio, N., Dario, S., Silvia, M., Nicola, G., Arnoldo, P., Andrea, B., Barbara, D., Stig, A.L., Guido, R., Marco, P.: Principal component analysis of FDG PET in amnestic MCI. Eur. J. Nucl. Med. Mol. Imaging 35(12), 2191-2202 (2008)

5. Grady, C.L., McIntosh, A.R., Beig, S., Keightley, M.L., Burian, H., Black, S.E.: Evidence from functional neuroimaging of a compensatory prefrontal network in Alzheimer's disease. J. Neurosci 23(3), 986-993 (2003)

6. Haller, S., Nguyen, D., Rodriguez, C., Emch, J., Gold, G., Bartsch, A., Lovblad, K., Giannakopoulos, P.: Individual prediction of cognitive decline in mild cognitive impairment using support vector machine-based analysis of diffusion tensor imaging data. J. Alzheimers Dis. 22(1), 315-527 (2010)

7. Leemans, A., Jeurissen, B., Sijbers, J., Jones, D.K.: ExploreDTI: A graphical toolbox for processing, analyzing, and visualizing diffusion MR data. In: 17th Annual Meeting of Intl. Soc. Mag. Reson. Med., Hawaii, USA, p. 3537 (2009)

8. Machulda, M.M., Senjem, M.L., Weigand, S.D., Smith, G.E., Ivnik, R.J., Boeve, B.F., Knopman, D.S., Petersen, R.C., Jack Jr., C.R.: Functional MRI changes in amnestic and non-amnestic MCI during encoding and recognition tasks. J. Int. Neuropsych. Soc. 15(3), 372-382 (2009)

9. McEvoy, L.K., Fennema-Notestine, C., Roddey, J.C., Hagler Jr., D.J., Holland, D., Karow, D.S., Pung, C.J., Brewer, J.B., Dale, A.M.: Alzheimer disease: Quantitative structural neuroimaging for detection and prediction of clinical and structural changes in mild cognitive impairment. Radiology 251, 195-205 (2009)

10. Sokolova, M.V., Japkowicz, N., Szpakowicz, S.: Beyond accuracy, F-score and ROC: A family of discriminant measures for performance evaluation. In: Sattar, A., Kang, B.-h. (eds.) AI 2006. LNCS (LNAI), vol. 4304, pp. 1015-1021. Springer, Heidelberg (2006)

11. Tzourio-Mazoyer, N., Landeau, B., Papathanassiou, D., Crivello, F., Etard, O., Delcroix, N., Mazoyer, B., Joliot, M.: Automated anatomical labeling of activations in SPM using a macroscopic anatomical parcellation of the MNI MRI single-subject brain. Neuroimage 15, 273-289 (2002)

12. Van Dijk, K.R.A., Hedden, T., Venkataraman, A., Evans, K.C., Lazar, S.W., Buckner, R.L.: Intrinsic functional connectivity as a tool for human connectomics: Theory, properties and optimization. J. Neurophysiol 103, 297-321 (2010)

13. Wang, Z., Jia, X., Liang, P., Qi, Z., Yang, Y., Zhou, W., Li, K.: Changes in thalamus connectivity in mild cognitive impairment: Evidence from resting state fMRI. Eur. J Radiol. (2011) 